\title{
One-Handed Thumb Use on Smart Phones by Semi-literate and Illiterate Users in India
}

\author{
Dinesh Katre \\ Centre for Development of Advanced Computing (C-DAC), Pune, India \\ dineshecdac.in
}

\begin{abstract}
There is a tremendous potential for developing mobile-based productivity tools and occupation specific applications for the semi-literate and illiterate users in India. One-handed thumb use on the touchscreen of smart phone or touch phone is considered as an effective alternative than the use of stylus or index finger, to free the other hand for supporting the occupational activity. In this context, usability research and experimental tests are conducted to understand the role of fine motor control, usability of thumb as the interaction apparatus and the ergonomic needs of users. The paper also touches upon cultural, racial and anthropometric aspects, which need due consideration while designing the mobile interface. Design recommendations are evolved to enhance the effectiveness of one-handed thumb use on smart phone, especially for the benefit of semi-literate and illiterate users.
\end{abstract}

Keywords: Smart phone, touchscreen interface, productivity tools, thumb, interaction, stylus, usability, fine motor control, ergonomics, cultural and racial factors.

\section{Introduction}

\subsection{The Indian Context}

Presently, India has approximately 286 million mobile subscribers [11] and this number is likely to grow up to 560 million by 2012 [12]. This growth will encompass a significant portion of semi-literate and the 400 million illiterate population of India [20]. Telecom infrastructure in India is expanding its reach rapidly [3]. People from all walks of life are beginning to use smart phones and touch phones with the touchscreen interface. The cell phone markets in developed nations are getting saturated. As a result, the cell phone makers have to drop prices, and come up with ultralow-cost mobile phones for the markets in less-developed countries like India and China [15].

With the enhanced capabilities and rich features of such mobile phones, it has become possible to use them for a variety of personal and professional tasks [14].

Presently, the smart phones as designed to suit the needs of affluent users. But there is a huge opportunity for designing innovative mobile applications for an entirely new segment of users like fishermen, farmers, carpenters, electricians, 
fabricators, vegetable merchants, shopkeepers, drivers, transport managers, traffic controllers, factory workers, building contractors and even the housewives in India. Furthermore, many other field occupations can be considered. Mobile based productivity tools and applications can be developed to facilitate the activities like task planning, scheduling, estimation, order booking, accounting, management, etc.

When it comes to spending, there is a difference in the requirements of affluent smart phone users and the potential semi-literate, illiterate users.

Table 1. The requirements of smart phone users

\begin{tabular}{ll}
\hline Affluent Users & Semi-literate / Illiterate Users \\
\hline Feature richness & Minimalist design \\
Diverse applications & Productivity tools \\
Self-sufficient device & Complementing \\
Status indicator & Problem solver \\
Work and Entertainment & Work and entertainment \\
Self-indulging & Field attention \\
Cost is no barrier & Cost effective \\
\hline
\end{tabular}

Proposed productivity tools for semi-literate and illiterate users should be designed to require minimum text input like numeric data. Most of it would involve selection from a list of pre-defined options, checking and unchecking and iconic buttons. Therefore, the success of proposed touchscreen based mobile applications will primarily depend on the usability of the interaction apparatus and user interface design.

\subsection{The Choice of Interaction Apparatus}

\section{Unsuitability of stylus and index finger}

PDA phones need the stylus for interaction. iPhone requires the use of index finger for interaction. Our hypothesis is that stylus is more suitable for users with good fine motor control, which is developed out of writing practice. Hand-eye coordination and dexterity are the other essentials for using the stylus. The semi-literate and illiterate users may not have these skills. Interaction using stylus and index finger pose similar disadvantage of engaging both the hands and attention of user. It constrains the movement and field activity of users. Coordination between various elements like both the hands (one hand holding the smart phone and the other with its index finger or holding the stylus), eyes, attention and fieldwork can be very demanding. This approach has many overheads, which need to be reduced. Use of stylus or index finger does not seem like a suitable proposition for semi-literate and illiterate users, as they need to do lot of field work and physical activity. Engaging both hands for mobile interaction is against the objective of proposed mobile-based productivity tools and applications. Higher productivity can be achieved by providing a solution that maximizes the use of available resources and minimizes the overheads.

\section{Advantages of one handed thumb use}

In this situation, one-handed thumb use on the touchscreen of mobile device seems more suitable to allow the field activity. It can truly help in boosting their productivity. 
One-handed thumb use on touchscreen can benefit the users by freeing a hand for variety of physical and attentional demands [14]. It will allow the users to hold things or perform some activity using one hand, and operate the mobile device using the other. Thumb is well known as the opposing finger, rapid finger, the most flexible and dynamic of all the fingers. The research on thumb kinematics has presented the challenges involved in modelling the intricacy of thumb movements [16]. Therefore, a focused usability study of one-handed use of thumb on smart phones by semi-literate and illiterate users is essential. It will help in understanding the strengths and limitations of thumb as the apparatus for interacting with smart phones. It will also provide useful insights and design directions.

\subsection{Varying Characteristics of Thumb}

\section{Physical, cultural and racial characteristics}

Use of stylus insulates a whole lot of physical, cultural and racial issues, which otherwise begin to surface, especially while using the thumb as apparatus for interaction. Anthropologists have already presented the heterogeneous racial characteristics and differences in the anatomical proportions of human beings belonging to different races [4]. Singh et al. have analyzed and shown the effect of habitual activities on body proportions (including the shapes and sizes of hands) of traditional occupational groups [19]. Anthropometric data clearly indicates varied average heights of males and females from different countries and races. Average heights of Indian male and female are $165.3 \mathrm{~cm}$. Average heights of white American male and female are 178.2 $\mathrm{cm}$ and $164.1 \mathrm{~cm}$ respectively [23].

Therefore, proportionately the size and shape of hands and fingers also change from race to race and country to country.

\section{Ergonomic suitability}

A recent study has revealed thumb related Repetitive Stress Injuries (RSI) among the mobile workers who extensively used BlackBerry [21]. In this context, it becomes very important to study the ergonomic aspects of thumb as the apparatus for interaction and the design of user interface. The RSI continue to persist despite several ergonomic studies to design thumb keyboard [21].

\section{Objective of our usability study}

- Considering the multiple dimensions of one-handed use of thumb on smart phones, experimental tests are conducted with following objectives.

- Understand the difference between the fine motor control of educated, semi literate and illiterate users, while using the stylus and thumb on smart phone. Also find the common trends of proficiency and the usability problems faced by them.

- Study the usability, physical characteristics, qualitative, ergonomic aspects of thumb as the proposed apparatus for interacting with smart phones.

- Identify the factors involved in the use of thumb on touchscreen that can affect the quality of interaction. Use the findings of this study to enhance the user interface for thumb interaction.

This research is not intended to involve statistical study in its current scope. Our emphasis is more on collecting the qualitative and observational data. 


\section{Related Work}

The fundamental study on human motor systems in controlling the amplitude and movement by Fitts [5] has complemented our experimental work. We have adopted some aspects of the reciprocal taping apparatus given by Fitts, as part of the tests performed using thumb and stylus.

Karlson et al. have presented thumb gesture based Scalable User Interface (ScUI) technique to support multiple devices with different resolutions and aspect ratios [8]. Fisheye and pure zoom techniques are used over multiple applications for magnifying the user interface. Thumb gestures also require certain amount of fine motor control. Our study includes certain tests to find out the fine motor skills of semi-literate and illiterate subjects with respect to mobile use.

Wu et al. have carried out the performance study on touch-pen sizes in three screen tasks. They used 12 different touch-pens varying in length and diameter to perform pointing, clicking, writing and drawing tasks on the tablet PC [22]. This study has shown that the quality of performance can vary depending on the length and the diameter of touch-pen. We found this research very useful, as it creates a ground for conducting similar research on the use of thumb for interacting with smart phones. The length, diameter and shape of thumb also vary from user to user, which impacts the precision and overall quality of interaction.

Parhi et al. have conducted a study to identify optimal target size for one-handed thumb use on small touch screen devices [14]. It involved two phase experiments on discrete and serial tasks and hit response variability for different sizes of targets and locations. Their study recommends sufficiently large target size of $9.2 \mathrm{~mm}$ for discrete tasks and $9.6 \mathrm{~mm}$ for serial tasks for one-handed thumb use on touch screen handheld devices. These experiments were carried out on 20 right handed subjects in the age group of 19 to 42 years using HP iPAQ h4155 PDA.

These findings may be true for certain class of users only. In our study, the socicultural context is Indian and the targeted users are semi-literate and illiterate. Our focus is not on identifying the target size but on the usability of thumb as the apparatus for interaction. The experimental tests used in our study are meant to identify the fine more control and the natural movements of thumb.

Yunfei has carried out an ergonomic experiment for thumb keyboard design [24]. Balakrishnan el al. have investigated the effect of varying sizes of thumbs, while using the mobile keypad for texting [1]. Both of these experiments are complementing to our experiments, as they have covered some of the anatomical aspects thumb while evaluating the keyboard of mobile phones.

Gokturk et al. have analysed the index finger as a pointing device in comparison with mouse and joystick [6]. Apart from these, there have been several studies to provide tactile feedback for finger based text entry [2], to understand the problems involved in the bilingual keypad layout of mobile handsets [8] and to evolve guidelines for the button sizes $[18,13]$ for old persons $[10,19]$.

\section{User Categories}

The categories of users are broadly defined as under. 


\section{Semi-literate Users-}

- These are such people who have left the education half way due to economic reasons. But they are familiar with reading and writing.

- Their occupation is such that they seldom require to write or they do not require to write anything. Their writing skills have significantly eroded.

- Their occupation involves lot of physical activity and fieldwork.

- They are familiar with mobile phones.

\section{Illiterate Users-}

- These are such users, who have never been school. They do not have reading or writing skills.

- They can understand English numbers. They have learnt basic addition and subtraction of numbers through financial transactions, as the values on Indian currency notes and coins are mentioned in English.

- Their fine motor control (specifically the skill that is developed out of writing practice) is not well developed due to lack of education.

- Their occupation involves major physical activities and fieldwork.

- They are aware of mobile phones but they may not have used it.

We focus on such semi-literate and illiterate users, whose occupation / business justifies the use of mobile-based productivity tools in terms of its complexity, revenue, activity, etc. Sample occupations are enlisted earlier in point 1.1. For the purpose of comparison, the characteristics of educated users are also considered.

\section{Educated Users-}

- They have completed education till at least graduation level. They are proficient in reading and writing.

- Their occupation is such that they need to read and write quite frequently.

- Their fine motor skills are well cultivated.

- They are very familiar with mobile phones.

Following short forms are introduced while discussing our experimental testsEducated Subjects (ES), Semi-literate Subjects (SLS), Illiterate Subjects (IS).

\section{Participants}

Our experiments were performed on total 21 participants within the age group of 25 40 years. The details are as under-

- Semi-literate Subjects (SLS): 7

- $\quad$ Illiterate Subjects (IS): 7

- $\quad$ Educated Subjects (ES): 7

Above participants included females and left-handed subjects.

- Left-handed Subjects: 4

Educated: 1, Semi-literate: 2, Illiterate: 1

- Female Subjects: 4

Educated: 3, Semi-literate: 1, Illiterate: 0

The semi-literate and illiterate subjects included people with different professions like electrician, gardener, estate manager, farmer, driver and housewives.

\section{Test Apparatus}

Various PDA phones were observed such as HP IPAQ Pocket PC, HTC TYTN 4550, iPhone, etc. Out of these, HTC TYTN 4550 PDA phone with its stylus was chosen for 
testing. It was more suitable for testing due to smaller size of display (i.e. 2.8inch diagonally). HP iPAQ and iPhone displays are 3.5inch in size, which is quite large for one-handed thumb use. The specifications of HTC TYTN 4550 are given below-

Display: 2.8 inch, 240x320 QVGA TFT-LCD touchscreen

Operating System: Windows Mobile 6 Professional

Dimensions: $112 \mathrm{~mm}$ (L) x 59mm (W) x 19mm (T)

Our main intention of the study is to evaluate 'thumb' as the apparatus for interacting with the touchscreen and not to evaluate any specific model of PDA phone. Also, we do not intend to compare multi-touch or single touch aspects of touchscreens. Therefore, use of HTC TYTN 4550, as part of our experimentation, is only incidental. We chose it because of its smaller size, as that allows one to hold it in one hand and operate it using thumb.

\section{Design of Experimental Tests}

Agile approach to experimental testing is explored for identifying various usability issues centred around the use of thumb for touchscreen interaction. It meant incremental change in the test design or conceptualization of new test based on the findings and insights.

All experimental tests were designed using Adobe Flash CS 3 Professional and played through the PDA phone.

I. Initially, an experimental test was designed to evaluate the fine motor control of subjects. It involved multiple grids with varying sizes of buttons as shown in figure 1. The subjects had to click the buttons in serial order using stylus first and then the thumb. We kept on increasing the sizes of buttons until it improved the performance results using the thumb. This type of testing was helpful in checking the hit rate in one-minute duration for various subjects.

II. A separate test was designed for testing the accuracy of thumb clicking. In this, discrete clickable targets were presented in random locations on the screen of PDA phone. It helped in identifying the difference in the intended location of clicking and the actually clicked location. It also helped us in identifying the common patterns of clicking for every subject.

After observing the results of initial two types of testing, the next set of tests were evolved.

III. A piece of paper, matching the size of screen, was stuck on the mobile device. Thumb impressions were taken on this paper by applying ink to the tip of thumb. This test helped us in observing the contact area of thumb, its shape and angle, when it touched the screen. It also revealed the points on the tip of thumb and the screen locations, where the force is concentrated.

IV. Buttons were arranged in a curved line, instead of usual straight-line arrangement of buttons. This test was designed to find the comfort and ease of use while clicking. Elongated shape of buttons was also tested in a similar way.

Total 10 tests were evolved. Out of these initial 6 tests involved gradual increase in the buttons size. The tests were performed in a lab environment and in the sitting 
posture to minimize the complexities. Every subject was given enough time to practice before testing, until they felt comfortable with the medium.

Various lengths and shapes of thumbs, the use of thumb by different types of subjects on the PDA phone were minutely observed. The observations are insightful enough to seek design directions. The details of each experimental test are elaborated in the sections ahead.

\section{Experiments}

\subsection{Test of Fine Motor Control Using Stylus}

\section{Description of test}

As shown in figure 1. interactive applications containing varied sizes of grids with clickable buttons were prepared using Flash CS 3. Educated, semi-literate and illiterate subjects were asked to use the stylus for clicking the buttons provided in $28 \times 28$, $20 \times 20$ and 15x15 grids. Subjects were tested on each grid separately.

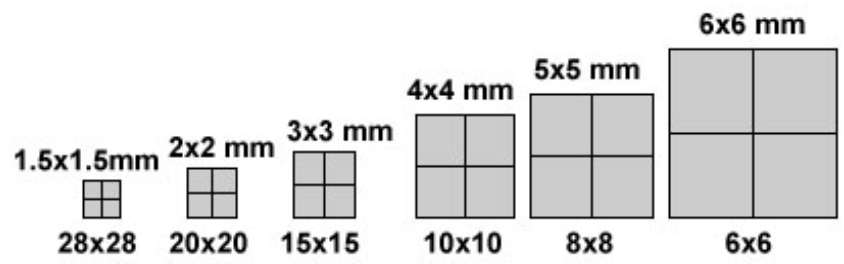

Fig. 1. Different sizes of grids with clickable buttons

The behaviour of flash application was designed as under-

- Each test was conducted for exactly one-minute duration in sitting posture. The timer began ticking after the first click of the button in the grid. The time-out was indicated by displaying a blue circle on the screen.

- The subjects were given sufficient time to practice, until they felt confident, before starting to test. Each subject was tested separately.

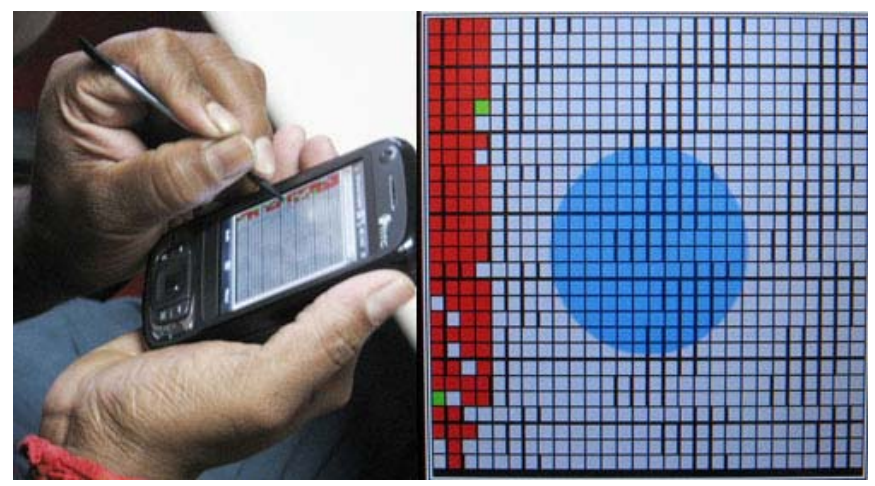

Fig. 2. PDA with $28 \times 28$ Grid 
- After clicking a button, it changed its color to red for feedback. Repeated click on the same button changed its color to green.

- The subjects were asked to click the buttons in serial order with a comfortable pace, without the permission to return if they skipped some buttons. They were instructed to click with proper concentration on the task, without skipping any buttons.

- In this manner each subject was asked to click sequentially, in different directions and in different parts of the screen, as shown in table 2.

- After the time-out, photographs of the last screen were taken for counting the total number of clicks and errors. The subjects were minutely observed when they performed the test.

Table 2. Test of sequential clicking in different directions

\begin{tabular}{ll}
\hline Part of PDA screen & Order of clicking \\
\hline Upper side of PDA screen & Left to Right (L-R) \\
& Right to Left (R-L) \\
Left side of PDA screen & Top to Bottom (T-B) \\
Right side of PDA screen & Top to Bottom (T-B) \\
Left side of PDA screen & Bottom to Top (B-T) \\
Right side of PDA screen & Bottom to Top (B-T) \\
Lower side of PDA screen & Left to Right (L-R) \\
& Right to Left (R-L) \\
\hline
\end{tabular}

\section{Objective of Test}

This test was designed with the following objectives.

1. Observe the difference between the fine motor control of educated, semiliterate and illiterate subjects in terms of the percentage of accurate clicks and errors (miss outs and repeated clicks).

2. Find out the subject's proficiency or lack of proficiency while clicking in different directions and different parts of the screen.

\section{Results and observations}

Initial tests on $28 \times 28$ grid with tiny buttons challenged the fine motor control and hand-eye coordination of subjects, as it required greater concentration of eyes on the target and consistent incremental movement of the lower arm. Miss-outs and repeated clicks on the same buttons represented the misjudgement of subjects. Subjects missed out some of the buttons while clicking, as their lower arm shifted a little more than necessary. After skipping a few buttons by mistake the subjects became extra careful while controlling the gradual movement of lower arm. This resulted in repeated clicks on same buttons, which is also counted as an error. We found higher frequency of such errors if subjects had poor fine motor control.

\section{Clicking in Left to Right order (Upper side of PDA screen)}

- While clicking from left to right direction, we found that the error rate of semi-literate and illiterate subjects was much higher than educated subjects. 
- The illiterate subjects became extremely conscious while holding the PDA and stylus in their shivering hands, and ended up clicking very slowly. This resulted in very less number of clicks.

- But the error rate of illiterate subjects became very high whenever they increased the speed of clicking as shown in graph 1 . Total number of clicks within one minute by an illiterate subject are less by $64 \%$, and that too with higher error rate, if compared with the educated subject.

- Educated subjects clicked more number of buttons speedily with few errors.

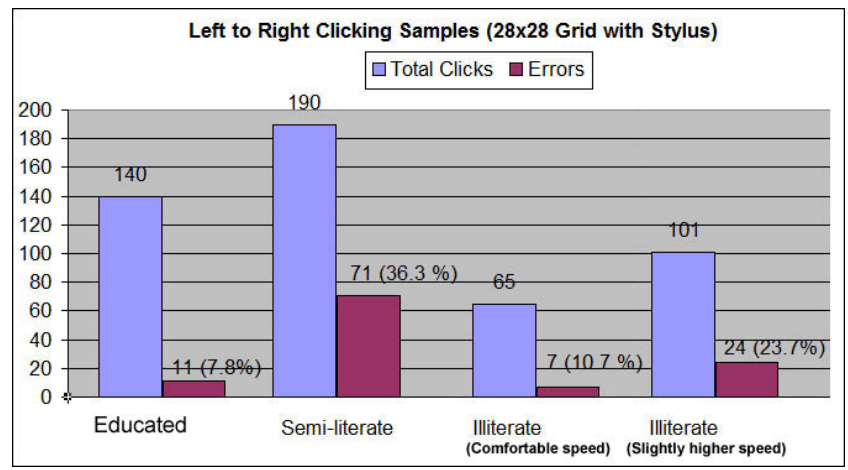

Graph 1. Comparison of left to right clicking performance between the subjects

\section{Dexterity}

- Right-handed subjects demonstrated greater proficiency while clicking the buttons in left to right order, if compared with their performance in right to left order.

- Left-handed subjects demonstrated greater proficiency while clicking the buttons in right to left order, if compared with their performance in left to right order.

- In case of right-handed illiterate subjects, their strength was not clearly visible in any of the tests. It showed lack of dexterity while using the stylus.

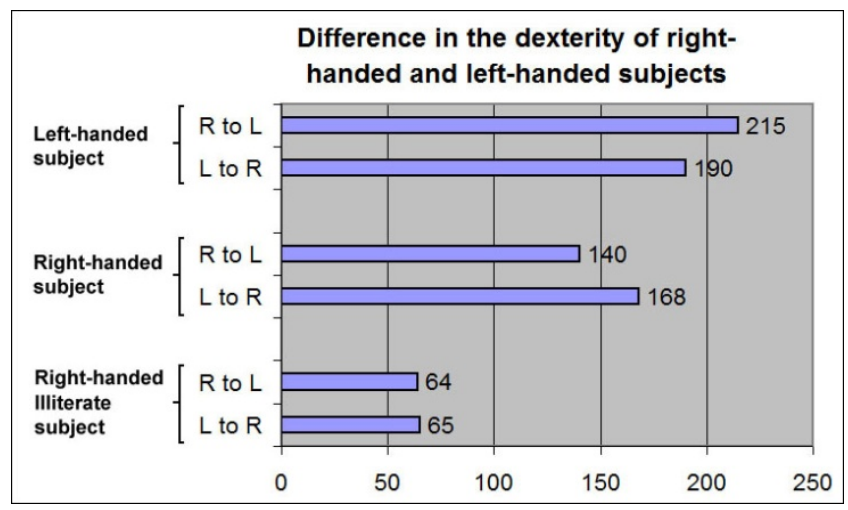

Graph 2. Difference in the dexterity of subjects (28x28 Grid) 


\section{Clicking in Left to Right order (Lower side of PDA screen)}

- All the subjects were not comfortable while clicking the buttons at the lower part of the PDA screen, as the hand does not get adequate support.

- On the contrary, while clicking in the upper portion of the screen, one was able to rest the hand on the edge of the PDA and hence their performance in this area was much better. presents the performance rating of a semi-literate subject.

\section{Clicking from the top to bottom and bottom to top}

- Top to bottom and bottom to top clicking sequences did not show any significant trend.

\section{Slippery and thin stylus}

- Figure 2. shows the photograph of how the illiterate subjects were holding the stylus in hand. The stylus was too thin and slipper for them to grip it properly between the fingers. It is also because they have seldom held the pen in hand for writing.

\section{Fatigue}

- We also found that subjects made more errors in the second half of the clicking tasks due fatigue.

\section{Performance on 20x20 and 15x15 grids}

- In the subsequent tests over bigger size of grids such as $20 \times 20$ and 15x15, the performance of subjects improved marginally.

This test revealed that the stylus wasn't really a good option for the semi-literate and illiterate subjects due to their lack of fine motor control. The tests hereafter were performed on $10 \times 10,8 \times 8$ and $6 \times 6$ grid using the thumb, as the button sizes were large enough to allow thumb clicking.

\subsection{Interaction Using the Thumb}

\section{Description of Test}

In this test, all subjects were asked to click using their thumb instead of the stylus. 10x10 grid with buttons admeasuring $4 \mathrm{~mm}$ by $4 \mathrm{~mm}$ was used for this test. As shown in table 2., we followed the same pattern of clicking on PDA screen. The functional behavior of the application and rules were same as earlier.

\section{Objective of test}

1. Find out whether the educated subjects are able to do better using the thumb, if compared with semi-literate and illiterate subjects. The intention was to see whether the fine motor control developed out of writing practice is helpful in thumb use.

2. Observe the error rate and compare it with subjects' performance using stylus.

3. Identify the areas on PDA screen where the subjects are more efficient and effective.

\section{Results and observations}

All categories of subjects made greater number of errors while performing the test using their thumb. The performance of educated subjects also declined significantly in terms of accuracy if compared with their performance using the stylus. 


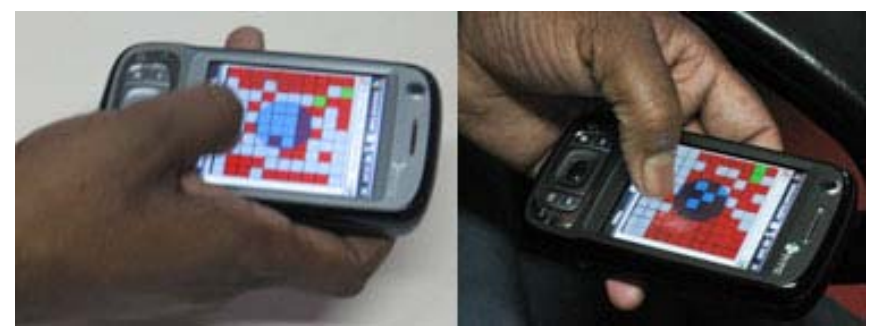

Fig. 3. Thumb clicking by right handed (IS) and left handed (SLS) on 10x10 grid

\section{Palm size and thumb length}

- Subjects with smaller palm and shorter length of thumb could not click the targets near the outside edge and upper left corner of the PDA screen. As they have to stretch the thumb which flattens the touch on the screen. Refer figure 6 .

- Subjects with bigger palm and long length of thumb could not click the targets near the inside edge and lower right or left corner of PDA screen respectively (based on whether they are right-handed or left-handed). Refer figure 3.

\section{Shape of thumb and fingernails}

- Subjects with blunt shape of thumb made more errors.

- Subjects with properly shaped and long fingernails were more accurate. All semiliterate and illiterate subjects that participated in our test had slightly long and rounded fingernails. Refer figure 3 .

- Educated subjects had short but properly shaped fingernails. They too were able to click properly.

- Small number of subjects with unkempt fingernails. They had to struggle a bit more for getting the proper touch on buttons.

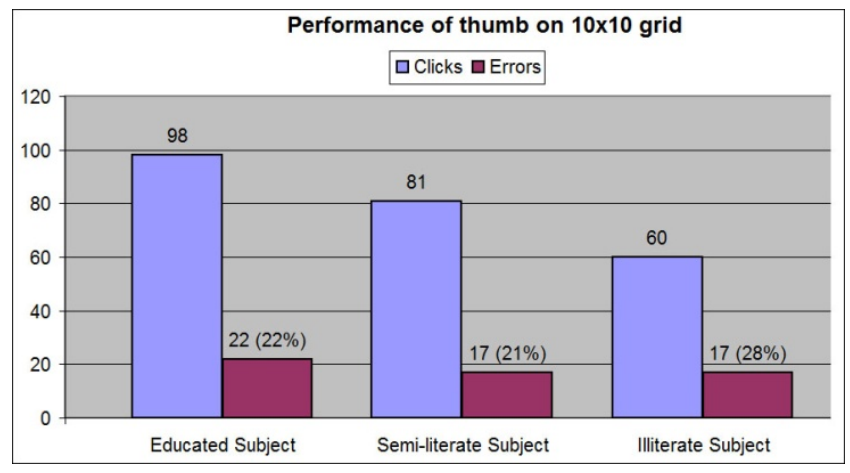

Graph 3. Performance using thumb on 10x10 grid

\section{Dexterity}

- As shown in graph 4. all categories of subjects did not demonstrate dexterity in terms of their right-handedness or left-handedness when they used their thumb, if 
compared with graph 2. Their clicking performance in left to right and right to left sequences is almost similar. It means the fine motor control developed out of writing practice wasn't helping the educated subjects as much as it did while using the stylus.

\section{Raised frame around the touchscreen}

- The subjects faced difficulty while clicking the buttons near the raised frame around the touchscreen. Unlike iPhone, many other smart phones have a raised frame around the screen. It is not recommendable for one-handed thumb use.

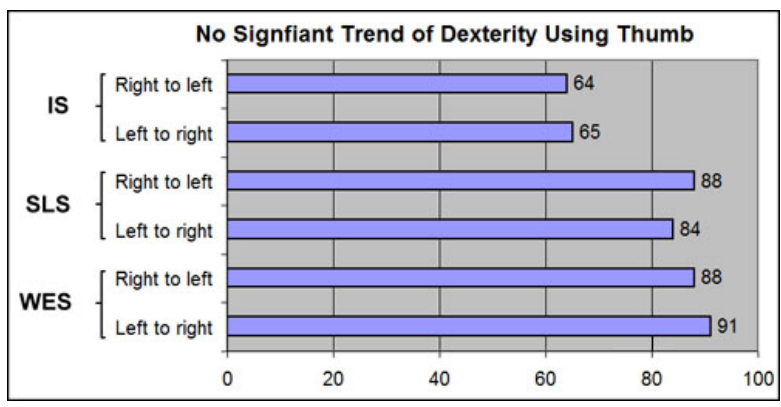

Graph 4. No sign of dexterity using thumb

\section{Good performance in $8 \times 8$ and $6 \times 6$ grids}

- Educated subjects and semi-literate subjects could easily click the buttons on $8 x 8$ grid with minor errors. Here the diagonal measurement of button was $7 \mathrm{~mm}$.

- But the illiterate subjects found it difficult and made many mistakes while clicking on $8 \times 8$ grid. They were more comfortable with $6 \times 6$ grid and made almost no errors. Here the diagonal measurement of the button was $9 \mathrm{~mm}$.

- Looking at the consistent trend of improvement throughout the testing, we believe that with more practice of thumb use, the semi-literate and illiterate subjects will be able do as good as educated users.

\subsection{Accuracy of Clicking Using Thumb}

\section{Description of test}

This test involved a grid of $15 \times 15$ clickable buttons. However, only the $5 \times 5$ grid is made visible to the subjects. Each box in the grid actually consists of $3 \mathrm{X} 3$ (total 9) clickable buttons, which are not outlined. One tends to perceive the group of 9 buttons as the single target to be clicked using thumb.

In this test each box appeared on the screen after 3 seconds and continued to remain there till end. Every time, the new box appeared in different and unpredictable location of screen. In this manner, the subjects were asked to click all 25 boxes. The subjects had to perform this experiment three times.

As each box is consisting of 9 clickable buttons. While clicking using the thumb, the area where maximum force was concentrated, the button underneath got clicked 
and changed its color to dark blue. When the box was hit exactly in the center, the button the in center of the box got clicked.

\section{Objective of test}

- Evaluate the preciseness of clicking using the thumb. Understand the reasons behind why the subject was unable click exactly in the center of target, repeatedly in certain locations.

- Find out whether there are any common patterns in the clicked points and locations on PDA screen.

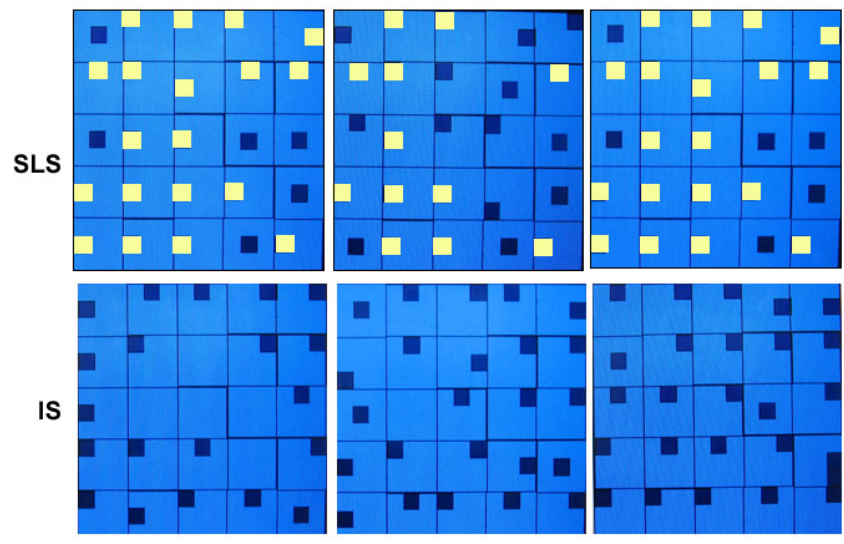

Fig. 4. Pattern of thumb clicking

\section{Observations \\ Pattern}

- We found that each person has a common pattern of clicking using the thumb, which consistently repeated.

- As shown in figure 4. (first row of three photographs) the pattern of clicking is indicated by highlighting the boxes in yellow color. Each time the subject has tried to click the box in its center but in certain locations (s)he has ended up clicking either on left side of the box or at the upper portion of the box. The second row of three photographs show the performance by an illiterate subject.

\section{Common miss outs}

Many times the subjects failed to click in the center of target although their intention was always to click in the center. It shows the unpredictability of the thumb as a pointing device.

\subsection{Thumb Impressions on PDA Screen}

\section{Description of test}

The earlier test showed that there is consistent pattern in the way the thumb touched the screen in different locations. Therefore, we decided to experiment with another test, in which ink was applied on the thumbs of subjects. A piece of paper exactly 
matching the screen size was stuck on the PDA. The subjects were asked to touch different locations on the paper, while holding the device in one hand. Thumb impressions that appeared on paper were used for analysis.

\section{Objective of test}

- Observe the contact area of thumb in different locations of screen; and its changing shape and angle based on the length of the thumb.

\section{Observations}

\section{Flat and pointed finger touch}

- During one-handed thumb use on PDA screen, certain locations are conveniently within the reach of the thumb; and for certain locations the thumb has to be stretched.

- As shown in figure 5(i), as per the thumb impressions, the contact area of the thumb towards the lower right corner of the screen has become elongated and narrow. Whereas, the contact area of the thumb towards the top left corner and middle of the screen, is quite large and oval shaped.

\section{Stretching and shrinking for length adjustment}

- Figure 5(ii). shows that the thumb needs to be stretched to reach the locations A1, A2, A3 and B1 for those with shorter length of thumb. Such stretching can be stressful.

- Whereas, the thumb has be folded and shrunk in its length to reach the locations E3, E2, D3, D2, D1 and C1. Such shrinking can be stressful for those having long length of thumb.

- Locations like E1, D1, C1, C2, B2, B3 are comfortably reachable for most subjects.

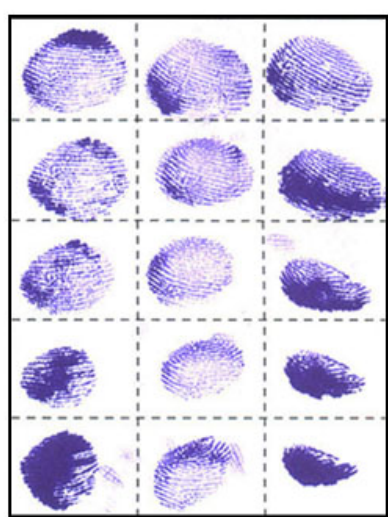

(i)

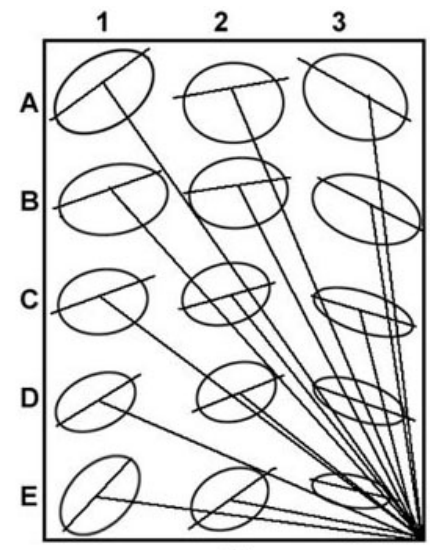

(ii)

Fig. 5. (i) Thumb impressions reveal the contact area in different locations of screen (ii) Asymmetric movement of thumb from lower right corner to all over the screen causing stressful joint movements due to length adjustment required for clicking the target 


\section{Thumb approach from a corner of PDA screen}

- It is most important to note that the thumb approaches the PDA screen from lower-right corner (if right-handed) and lower-left corner (if left-handed). This also results in asymmetric movements.

\section{Asymmetric movements}

- Thumb approach is from a corner of the screen, which causes the thumb to move in an asymmetric way. As shown in figure 5(ii), while moving from target A1 to A3 one has to gradually shorten the length of thumb.

- When using the stylus, its length remains constant while approaching any of the targets on the screen.

\section{Circular motion of thumb}

- The thumb has a natural circular movement centered around its joint that connects with the palm. But we forcibly move our thumb linearly in horizontal direction on the screen. It can stress the thumb joints and cause Repetitive Stress Injuries (RSI) in the long run.

- Also, the contact area of thumb is oval shaped. Then why should the buttons be rectangular or square shaped?

- As shown in figure 7., we can evidently notice circular wiper like movement of the thumb, which is natural.

- But contrary to this, the user interface, menus, and buttons are organized in linear order. It forces the thumb to be moved in straight line, against its natural circular motion.

\subsection{Physical Study of Thumb}

Some common observations are already mentioned in point 6.2. We measured the thumbs of subjects based on the length of its phalanges and the circumference of the distal phalange (tip of the finger) as shown in figure 6(i). Sample measurements are given in table 3 .

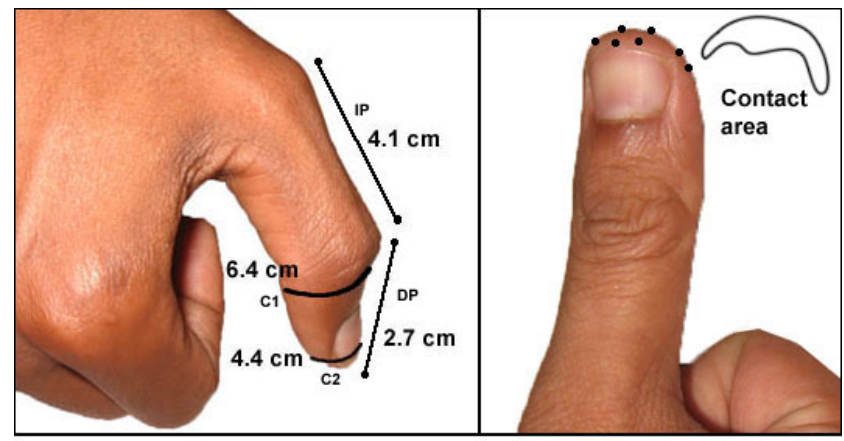

(i)

(ii)

Fig. 6. (i) Measurements of thumb (ii) Different contact points of thumb on the touch screen 


\section{Blunt distal phalange}

Even within the small sample of thumbs measured by us we found some exceptions where the circumference of the distal phalange (C2) was slightly larger by couple of millimeters than the other comparable thumbs. Refer table 3 . We found these subjects struggling a bit harder than others, while clicking the targets.

\section{Pointed Distal Phalange}

We also came across many subjects who had pointed thumbs, with significantly less circumference of the distal phalange.

\section{Asymmetrically distributed and shifting points of contact}

As shown in figure 6(ii) the tip of the thumb touches the screen at multiple points. Thumb is not a single point device like the stylus. Mostly the points of contact on the thumb move along the horizontal axis and they are distributed more on the right half of the tip for right-handed users.

\section{Impact of occupation}

Softness of the palm skin and the shape of fingernails also depend on the occupation of subjects e.g. farmers have rough hands. All educated subjects had softer hands if compared with the semi-literate and illiterate subjects.

Table 3. Sample measurements of thumb

\begin{tabular}{|c|c|c|c|c|c|c|c|}
\hline \multicolumn{4}{|c|}{ Female Thumbs } & \multicolumn{4}{|c|}{ Male Thumbs } \\
\hline IP & DP & $\mathrm{C} 1$ & $\mathrm{C2}$ & IP & DP & C1 & C2 \\
\hline 3.7 & 2.9 & 5.1 & 3.7 & 4.1 & 3.4 & 6.4 & 4.4 \\
\hline 3.5 & 2.7 & 5.2 & 3.4 & $\underline{3.9}$ & $\underline{3.5}$ & $\underline{6.3}$ & $\underline{4.7}$ \\
\hline$\underline{3.7}$ & $\underline{2.8}$ & $\underline{5.4}$ & $\underline{3.4}$ & $\underline{3.8}$ & $\underline{3.4}$ & $\underline{5.7}$ & $\underline{4.1}$ \\
\hline$\underline{3.7}$ & $\underline{2.9}$ & $\underline{5.6}$ & $\underline{3.9}$ & $\underline{3.8}$ & $\underline{3.4}$ & $\underline{6.1}$ & $\underline{3.9}$ \\
\hline
\end{tabular}

- Length of Intermediate Phalange (IP), Length of Distal Phalange (DP)

Measurements of distal phalange.

- Circumference 1 (C1).

- Circumference 2 (C2).

\section{Design Recommendations}

\section{Factors of thumb that impact the interaction}

Length of thumb, circumference of the tip of thumb, length and shape of fingernail, size of palm, the location of approach to the PDA screen (lower-left corner or lowerright corner), its contact point and contact area on the PDA screen.

\section{Why is thumb clicking ambiguous?}

The shifting contact point and varying contact area of thumb in terms of its shape, size and angle make it an ambiguous or imprecise apparatus for interacting with smart 
phones. The contact points shift and the contact area changes mainly because the thumb has to approach from the bottom corner of PDA screen.

\subsection{Improving the Thumb Precision through Design}

\section{Elongated shape of buttons}

It is noticeable that the multiple contact points on the tip of thumb, are mostly distributed in horizontal order, more towards the right half of the thumb for right-handed subjects. Therefore it is logical to have elongated shape for the buttons for better results.

As shown in figure 7, the curved arrangement of buttons was most convenient to all subjects. They unanimously felt that the circular motion of thumb was more natural than the straight-line movements on the screen.

\section{Capturing the size of contact area to decide the target size}

Universal and fixed target size may become too small or too large and not usable for many users. Therefore, it will be ideal to intelligently capture the size of contact area of thumb (location wise) for every user and then adjust the target sizes. This type of personalization may be essential at least for the productivity tools.

\section{Selectable user interface layouts}

Selectable user interface layouts should be provided for left-handed and right-handed users. They are very important in the context of one-handed thumb use, as the reachable locations on touchscreen are different for both types of users.

\section{Inclined screen for center alignment with thumb}

As shown in figure 8 , if we consider the angular position of the thumb, struggling to use it over a perpendicular screen can be definitely difficult and stressful. The inclined screen seems like an ergonomic proposition for one handed thumb use. It can bring certain amount of symmetry to the movement of the thumb. If the screen is positioned at an angle, in proper alignment with the thumb, the curved arrangement buttons is not necessary.

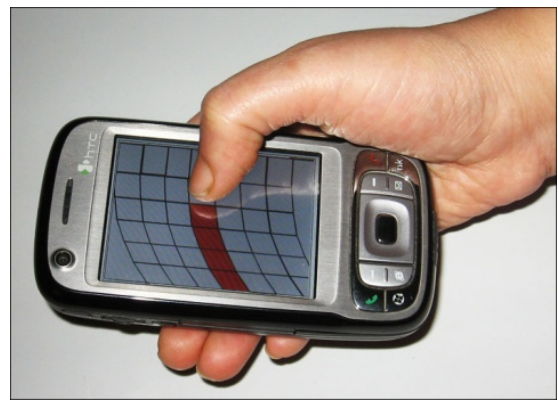

Fig. 7. Curved arrangement of buttons

\section{Size of screen and the device}

As shown in figure 8 the size of screen should ensure reachability of thumb in all locations. The raised frame around the screen should be avoided. The width of the device should be such that it allows one to properly hold it in hand. 


\section{Improved predictability and thumb control for the users}

If the thumb can approach the PDA screen from bottom-centre of PDA screen as shown in figure 7., the contact areas and contact points will be symmetrically distributed and it will become more controllable for the users for better precision.

\section{Reduced stress injuries}

The thumb movements will become natural, easy and ergonomically suitable. It will reduce the stretching and shrinking of the thumb joints at odd angles.

On the whole, thumb usability can be enhanced to a great extent if abovementioned design enhancements are incorporated.

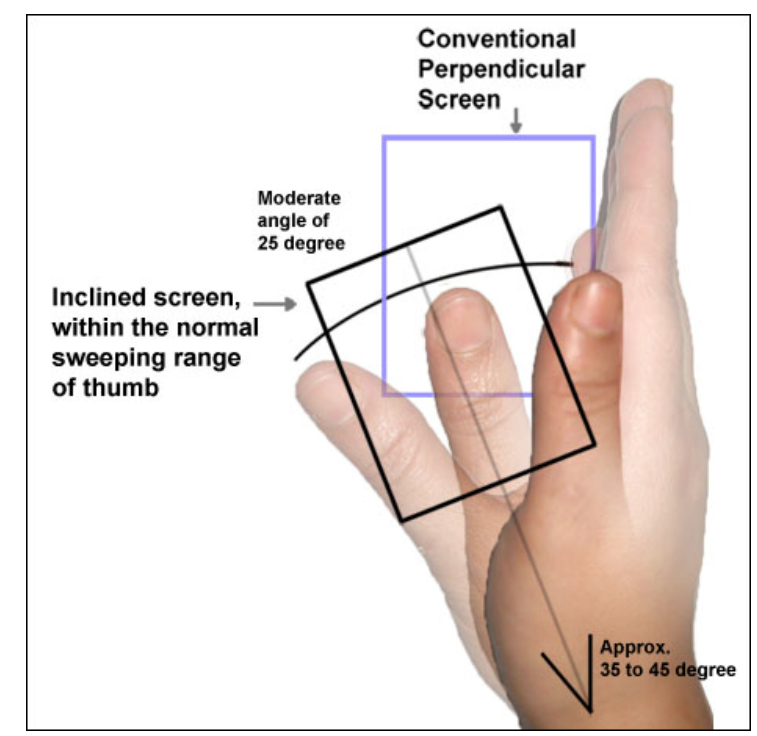

Fig. 8. Natural angular position of thumb and the proposed screen alignment

\section{Discussion}

The test of fine motor control using the stylus evidently shows that it gives greater advantage to educated subjects, if compared with the semi-literate and illiterate subjects. It is mainly because educated subjects have good writing practice.

The fine motor control developed through writing practice is marginally helpful while using the thumb for interaction. The other important observation is that there isn't contrasting difference between the performances of educated, semi-literate and illiterate subjects, when they used their thumb for clicking. This is primarily because; it is natural for all human beings to use their thumb quite proficiently.

We found that each person has a unique pattern of clicking using the thumb, which consistently repeats. This happens primarily because the thumb approaches the screen from a certain location and it has a fixed length and shape. One tends to misjudge the target because of the shape of thumb and its angular approach to the screen. 
Constant activity of stretching and shrinking of the thumb and angle adjustment to get the proper touch point on the screen, can put major stress on the thumb joints. Thumb kinematics [16] is really put to test when we use our thumb on the touch screen of smart phone.

Although we found that semi-literate and illiterate subjects could easily click the targets in $8 \times 8$ and $6 \times 6$ grid respectively, it is difficult identify one size of buttons, which will suit all. The suitable button size i.e. $9 \mathrm{~mm}$ found out by us, is smaller than what is recommended by Parhi et al. [14].

The anthropometric data of every country shows different anatomical proportions and average heights [23]. Universal size of button can be too large for some users or too small for some. Therefore, it would be ideal to intelligently capture the contact area of thumb in different locations of screen and adjust the button sizes accordingly.

The multiple contact points on the tip of thumb are spread horizontally, and more on the right half of the thumb for right-handed users. It shows asymmetry and imbalance due to angular approach of the thumb. It indicated that elongated shape of buttons improve the performance.

To remove the asymmetry and imbalance in the position and circular motion of thumb, it is suggested to incline the screen, so that the thumb can approach the touchscreen from its center of the bottom edge. It will help in reducing the stress and simplifying the kinematic adjustments [16] required for clicking various targets.

\section{Conclusion}

One-handed use of thumb as the apparatus for interacting with smart phone is recommendable for semi-literate and illiterate users. Their lack of fine motor control (skills developed out of writing practice) does not impact their use of thumb on the touchscreen. The design recommendations, which have emerged out of the finding of usability study, need to be incorporated to further improve the effectiveness of one-handed thumb use. With this, it will be possible to tap the potential market for mobile-based productivity tools and applications.

\section{References}

1. Balakrishnan, V., Yeow, P.: A Study of the Effect of Thumb Sizes on Mobile Phone Texting Satisfaction. Journal of Usability Studies 3(3), 118-128 (2008)

2. Brewster, S., Chohan, F., Brown, L.: Tactile Feedback for Mobile Interactions. In: Proc. CHI, pp. 159-162 (2007)

3. China and India Lead the Worldwide Telecom Carrier Capex and Revenue Growth, Celluar-news (July 2008), http: / / www. cellular-news.com/story/32251.php

4. Cavalli-Sforza, L.: L. Origin and Differentiation of Human Races. Proceedings of the Royal Anthropological Institute of Great Britain and Ireland 1972, 15-25 (1972)

5. Fitts, P.M.: The information capacity of the human motor system in controlling amplitude of movement. Journal of Experimental Psychology 47, 381-391 (1954)

6. Gokturk, M., Sibert, J.: An Analysis of Index Finger as a Pointing Device. In: Proc. CHI, pp. 286-287 (1999) 
7. Hoggan, E., Brewster, S., Johnston, J.: Investigating the effectiveness of tactile feedback for mobile touchscreens. In: Proc. Conference on Human Factors in Computing Systems, pp. 1573-1582 (2008)

8. Karlson, A.K., Bederson, B.B., SanGiovanni, J.: AppLens and LaunchTile: two designs for one-handed thumb use on small devices. In: Proc. CHI 2005, pp. 201-210. ACM Press, New York (2005)

9. Katre, D.: Cross-cultural Usability of Bilingual Mobile Phones. In: Proc. Indo-Danish Symposium on Cultural HCI, India (2006)

10. Kurniwiawan, S.: Mobile Phone Design for Older Persons. ACM Interactions XIV 4, 24 25 (2007)

11. India And Pakistan Gearing Up For Mobile Data Revenues (July 2008), http: //trendsniff.com/2008/07/31/

india-and-pakistan-gearing-up-for-mobile-data-revenues /

12. Monthly Economic Analysis, Fortune 2008, A Report published by Ministry of External Affairs, Government of India, pp. 5-6 (May 2008)

13. Mizobuchi, S., Mori, K., Ren, X., Yasumura, M.: An empirical study of the minimum required size and the number of targets for pen on the small display. In: Paternó, F. (ed.) Mobile HCI 2002. LNCS, vol. 2411, pp. 184-194. Springer, Heidelberg (2002)

14. Parhi, P., Karlson, A., Benderson, B.: Target Size Study for One-handed Thumb Use on Small Touchscreen Devices. In: Proc. Conference on Mobile HCI, pp. 203-210 (2005)

15. Sandova, G.: Falling revenue for cell phone maker (iSuppli Report), CNET News.Com (January 2006),

http: //news.cnet.com/Falling-revenue-for-cell-phone-makers / 2100-1039_3-6026127.html

16. Santos, V.J., Valero-Cuevas, F.J.: Thumb kinematics with non-orthogonal and nonintersecting axes of rotation may be necessary to predict realistic isometric thumbtip forces in multiple directions. In: Proceedings of the 28th Annual Meeting of the American Society of Biomechanics, Portland, OR (September 2004)

17. Sears, A., Revis, D., Swastiki, J., Crittenden, R., Shneiderman, B.: Investigating Touchscreen Typing: The effect of Keyboard Size on Typing Speed. Behavior and Information Technology 12(1), 17-22 (1993)

18. Siek, K., Rogers, Y., Connelly, K.: Fat Finger Worries: How Older and Younger Users Physically Interact with PDAs, Interact (2005)

19. Singh, A.P., Singh, S.P.: Impact of Habitual Physical Activity on the Human Body Proportions: A Comparative Study of Some Traditional Groups. Journal of Human Ecology 22(3), 271-275 (2007)

20. The World Factbook, India, https://www.cia.gov/library/publications/the-worldfactbook/print/in.html

21. Worthen, B.: Are Mobile Workers at Risk of BlackBerry Thumb? Business Technology, Wall Street Journal (June 2008)

22. Wu, F.G., Luo, S.: Performance study on touch-pens in three screen tasks. Applied Ergonomics, 149-158 (2006)

23. Human Height, Wikipedia, http://en.wikipedia.org/wiki/Human_height

24. Yunferi, X.: Ergonomic Experiment for Thumb Keyboard Design. In: IEEE CAIDCD, pp. $1-5(2006)$ 